\title{
Immediate versus Delayed Sarcoma Reconstruction: Impact on Outcomes
}

\author{
Kyle J. Sanniec, ${ }^{1}$ Cristine S. Velazco, ${ }^{2}$ Lyndsey A. Bryant, ${ }^{3}$ Nan Zhang, \\ William J. Casey III, ${ }^{3}$ Raman C. Mahabir, ${ }^{3}$ and Alanna M. Rebecca ${ }^{3}$ \\ ${ }^{1}$ Department of Plastic Surgery, University of Texas Southwestern, 1801 Inwood Road, Dallas, TX 75390, USA \\ ${ }^{2}$ Division of General Surgery, Mayo Clinic in Arizona, 5777 E. Mayo Boulevard, Phoenix, AZ 85054, USA \\ ${ }^{3}$ Division of Plastic and Reconstructive Surgery, Mayo Clinic in Arizona, 5777 E. Mayo Boulevard, Phoenix, AZ 85054, USA \\ ${ }^{4}$ Division of Health Sciences Research, Mayo Clinic in Arizona, 13400 East Shea Boulevard, Scottsdale, AZ 85259, USA
}

Correspondence should be addressed to Alanna M. Rebecca; rebecca.alanna@mayo.edu

Received 24 March 2016; Accepted 16 June 2016

Academic Editor: Mustafa Benekli

Copyright (C) 2016 Kyle J. Sanniec et al. This is an open access article distributed under the Creative Commons Attribution License, which permits unrestricted use, distribution, and reproduction in any medium, provided the original work is properly cited.

\begin{abstract}
Background. Sarcoma is a rare malignancy, and more recent management algorithms emphasize a multidisciplinary approach and limb salvage, which has resulted in an increase in overall survival and limb preservation. However, limb salvage has resulted in a higher rate of wound complications. Objective. To compare the complications between immediate and delayed (>three weeks) reconstruction in the multidisciplinary limb salvage sarcoma patient population. Methods. A ten-year retrospective review of patients who underwent sarcoma resection was performed. The outcome of interest was wound complication in the postoperative period based on timing of reconstruction. We defined infection as any infection requiring intravenous antibiotics, partial flap failure as any flap requiring a debridement or revision, hematoma/seroma as any hematoma/seroma requiring drainage, and wound dehiscence as a wound that was not completely intact by three weeks postoperatively. Results. 70 (17 delayed, 53 immediate) patients who underwent sarcoma resection and reconstruction met the inclusion criteria. Delayed reconstruction significantly increased the incidence of postoperative wound infection and wound dehiscence. There was no difference in partial or total flap loss, hematoma, or seroma between the two groups. Discussion and Conclusion. Immediate reconstruction results in decreased wound complications may reduce the morbidity associated with multidisciplinary treatment in the limb salvage sarcoma patient.
\end{abstract}

\section{Introduction}

Sarcoma remains a rare malignancy and accounts for less than $1 \%$ of all newly diagnosed cancers $(\sim 11,000$ new diagnoses a year in the United States) [1]. While early descriptions of treatment were focused on limb amputation, more recent management algorithms emphasize a multidisciplinary approach and limb salvage when feasible. The result of this evolution in care is increased overall survival and limb preservation [2], but the tradeoff being a higher rate of wound complications [3-5]. In exploring the etiology of these wound complications, our group uncovered a potential benefit in immediate reconstruction following sarcoma resection [6]. In an effort to further clarify the effect, we designed this study to compare the complications between immediate and delayed reconstruction in the limb salvage sarcoma patient population.

\section{Methods}

With IRB approval, a ten-year retrospective review of all patients who underwent sarcoma resection at our institution was performed. Patients with defects closed primarily were excluded from the study. The intervention compared was the timing of the reconstruction: delayed versus immediate. Delayed reconstruction was defined as any reconstruction occurring three weeks after primary oncologic resection, and immediate reconstruction was defined as any reconstruction within a three-week timeframe. Three weeks was chosen as the delayed reconstruction period, as patients had their first postoperative visit with orthopedics following oncologic resection; if wound complications were noted at that time, plastic and reconstructive surgery was consulted. Minimal dressings and debridement were used in the intervening time 
frame prior to delayed reconstruction. Demographic information and patient comorbidities, including obesity, peripheral vascular disease, coronary artery disease, diabetes, and active smoking or alcohol abuse, were collected. Neoadjuvant and intraoperative radiation and respective dosing were also recorded. The primary outcome of interest to the multidisciplinary team was wound complication in the postoperative period. To minimize observer, reporting, and recall bias, we defined infection as any infection requiring intravenous antibiotics, partial flap failure as any flap requiring a debridement or revision, hematoma/seroma as any hematoma/ seroma requiring drainage, and wound dehiscence as a wound that was not completely intact by three weeks postoperatively.

\section{Statistical Analysis}

Patients' demographics, diagnosis, chemotherapy, radiation, and additional health problems were summarized overall and compared between delayed and immediate reconstruction groups. Fisher's exact test was used for categorical variable comparisons, and two-sample $t$-test was used for continuous variable comparisons. Fisher's exact test was used to compare the outcomes between delayed and immediate reconstruction groups.

\section{Results}

A total of 70 (17 delayed, 53 immediate) patients who underwent sarcoma resection and reconstruction met the inclusion criteria. The most common pathologic diagnosis was myxofibrosarcoma in both reconstructive groups; for complete details see Table 1 . The median age of the patient population was 66.5 (range: 17-94) years, and 62.9\% were female. Obesity was the only significant comorbidity ( $p=$ 0.006) (Table 1). For type of flap, refer to Table 2.

Radiation was delivered to both groups (Table 1). 82.4\% of the delayed group received neoadjuvant radiation, whereas $64.2 \%$ of the immediate group did ( $p=0.2324)$; of those patients who received radiation, median dose was $50.4 \mathrm{gy}$ and 50.0 gy $(p=0.3955)$, respectively. $52.9 \%$ of the delayed group received intraoperative radiation, whereas $30.2 \%$ of the immediate group did ( $p=0.1443$ ); of those patients who received intraoperative radiation, median dose was $12.5 \mathrm{gy}$ in both groups $(p=0.5157)$.

Among patients who had delayed reconstruction, there were significantly more patients with wound infections $(n=$ $8,47.1 \%)$ and wound dehiscence $(n=11,64.7 \%)$ compared to patients who had immediate reconstruction (wound infection: $n=5,9.4 \%, p=0.0016$; wound dehiscence: $n=16$, $30.2 \%, p=0.0203)$. There was no difference between partial or total flap failure, hematoma, and seroma (Table 3).

\section{Discussion}

The reconstruction of sarcoma defects continues to represent a perfect storm for the plastic surgeon: a large tissue defect in a radiated field in a patient who has undergone chemotherapy. The most conspicuous finding of this study is that immediate reconstruction appears to result in a decreased rate of wound complications. This supports our previous work [6] on the importance of early plastic surgical intervention and reconstruction. Nearly $50 \%$ of the patients undergoing delayed reconstruction were diagnosed with an infection requiring intravenous antibiotics. Immediate reconstruction with vascularized soft tissue introduces healthy, nonradiated tissue, increases local blood flow, increases bacterial clearance, and consequently decreases infection rates.

Previous studies $[7,8]$ have demonstrated an infection rate from 15 to $30 \%$ in sarcoma patients, and in our study infection was seen in $47 \%$ of delayed reconstruction patients. This would suggest that there might be a role for prophylactic antibiotics for sarcoma patients who are undergoing delayed reconstruction. Interestingly, there are no current guidelines for prophylactic antibiotic use in this setting. Our results may also provide insight for those patients in whom immediate reconstruction is not an option.

There was no significant difference when evaluating partial or total flap loss between reconstructive groups. However, the only flap loss seen was in the immediate reconstructive group. While flap failure rate was low (5.7\%), we hypothesize that this may be due to the defect size caused by oncologic resection. Orthopedic surgeons may have more readily involved the reconstructive team when planning for a large resection that would require flap coverage. Lohman et al. observed this as well when comparing primary versus flap reconstruction. They hypothesize that filling the dead space created by the defect with well vascularized tissue may prevent wound complications when tissue pliability is lost from radiation or the immune system is compromised from comorbidities such as diabetes [9].

Neoadjuvant radiation can be associated with increased wound complications compared to postoperative radiation, which has more long-term morbidity and worse functional outcomes. However, these wound complications are often acute and manageable following neoadjuvant therapy [10]. Additionally, neoadjuvant therapy has been shown to have wound complication rates of $32 \%$ [11], and in a randomized trial, Baldini et al. found an overall complication rate of $52 \%$ following neoadjuvant radiation therapy among patients that required flap reconstruction [12]. Immediate reconstruction with vascularized tissue may provide improved wound healing through increased oxygenation and enhance antibacterial activity following radiation [13]. Our current work supports that decreased rates of wound complications are seen in immediate reconstruction following radiation.

The current study is a retrospective review and as such has limitations, including observer and selection bias. In an effort to minimize the effect of observer bias, we determined the outcomes of interest to be as objectively measured as possible. Initially, the orthopedic surgeons were performing the resections, intraoperative radiation, and the primary closure. As plastic surgeons made themselves available in sarcoma reconstruction, our group began doing more immediate reconstructions. The decrease in intraoperative radiation may be 
TABLE 1: Characteristics of the study population by reconstructive type.

\begin{tabular}{|c|c|c|c|c|}
\hline & Delayed $(N=17)$ & Immediate $(N=53)$ & Total $(N=70)$ & $p$ value $^{1}$ \\
\hline Age & & & & 0.3613 \\
\hline Mean (SD) & $59.8(16.9)$ & $64.3(18.0)$ & $63.2(17.8)$ & \\
\hline Median (range) & $66.0(17.0-81.0)$ & $67.0(22.0-94.0)$ & $66.5(17.0-94.0)$ & \\
\hline Sex, female (\%) & $11(64.7 \%)$ & $33(62.3 \%)$ & $44(62.9 \%)$ & 1.0000 \\
\hline Diagnosis, $n$ & & & & 0.4740 \\
\hline Myxofibrosarcoma & $5(29.4 \%)$ & $7(13.2 \%)$ & $12(17.1 \%)$ & \\
\hline Synovial sarcoma & $1(5.9 \%)$ & $5(9.4 \%)$ & $6(8.6 \%)$ & \\
\hline Leiomyosarcoma & $2(11.8 \%)$ & $4(7.5 \%)$ & $6(8.6 \%)$ & \\
\hline Liposarcoma & $2(11.8 \%)$ & $3(5.7 \%)$ & $5(7.1 \%)$ & \\
\hline Sarcoma NOS & $2(11.8 \%)$ & $4(7.5 \%)$ & $6(8.6 \%)$ & \\
\hline Myxoid chondrosarcoma & $1(5.9 \%)$ & $0(0.0 \%)$ & $1(1.4 \%)$ & \\
\hline Myxoid liposarcoma & $2(11.8 \%)$ & $2(3.8 \%)$ & $4(5.7 \%)$ & \\
\hline Fibrous histiocytoma & $0(0.0 \%)$ & $5(9.4 \%)$ & $5(7.1 \%)$ & \\
\hline Fibrosarcoma & $0(0.0 \%)$ & $4(7.5 \%)$ & $4(5.7 \%)$ & \\
\hline Pleomorphic sarcoma & $0(0.0 \%)$ & $7(13.2 \%)$ & $7(10.0 \%)$ & \\
\hline Spindle cell sarcoma & $1(5.9 \%)$ & $3(5.7 \%)$ & $4(5.7 \%)$ & \\
\hline Pleomorphic liposarcoma & $1(5.9 \%)$ & $0(0.0 \%)$ & $1(1.4 \%)$ & \\
\hline Dermatofibrosarcoma protuberans & $0(0.0 \%)$ & $1(1.9 \%)$ & $1(1.4 \%)$ & \\
\hline Angiosarcoma, high grade & $0(0.0 \%)$ & $1(1.9 \%)$ & $1(1.4 \%)$ & \\
\hline Giant cell rich extraosseous osteosarcoma & $0(0.0 \%)$ & $1(1.9 \%)$ & $1(1.4 \%)$ & \\
\hline Fibroblastic sarcoma & $0(0.0 \%)$ & $1(1.9 \%)$ & $1(1.4 \%)$ & \\
\hline Epithelioid angiosarcoma & $0(0.0 \%)$ & $2(3.8 \%)$ & $2(2.9 \%)$ & \\
\hline Osteosarcoma & $0(0.0 \%)$ & $1(1.9 \%)$ & $1(1.4 \%)$ & \\
\hline Neurofibrosarcoma & $0(0.0 \%)$ & $1(1.9 \%)$ & $1(1.4 \%)$ & \\
\hline Epitheloid sarcoma & $0(0.0 \%)$ & $1(1.9 \%)$ & $1(1.4 \%)$ & \\
\hline Neoadjuvant chemotherapy, $n$ & $8(47.1 \%)$ & $14(26.4 \%)$ & $22(31.4 \%)$ & 0.1381 \\
\hline Neoadjuvant radiation, $n$ & $14(82.4 \%)$ & $34(64.2 \%)$ & $48(68.6 \%)$ & 0.2324 \\
\hline Neoadjuvant radiation dosage, gy & & & & 0.1167 \\
\hline Mean (SD) & $39.9(19.7)$ & $30.0(23.3)$ & $32.4(22.7)$ & \\
\hline Median & $50.0(0.0-50.4)$ & $45.0(0.0-50.4)$ & $50.0(0.0-50.4)$ & \\
\hline Intraoperative radiation, $n$ & $9(52.9 \%)$ & $16(30.2 \%)$ & $25(35.7 \%)$ & 0.1443 \\
\hline Intraoperative radiation dosage, $g y$ & & & & 0.1240 \\
\hline Mean (SD) & $6.8(6.7)$ & $4.0(6.2)$ & $4.7(6.4)$ & \\
\hline Median (Range) & $10.0(0.0-15.0)$ & $0.0(0.0-17.5)$ & $0.0(0.0-17.5)$ & \\
\hline Obesity, $n$ & $8(47.1 \%)$ & $7(13.2 \%)$ & $15(21.4 \%)$ & 0.0060 \\
\hline Peripheral Vascular Disease, $n$ & $0(0.0 \%)$ & $1(1.9 \%)$ & $1(1.4 \%)$ & 1.0000 \\
\hline Coronary Artery Disease, $n$ & $1(5.9 \%)$ & $5(9.4 \%)$ & $6(8.6 \%)$ & 1.0000 \\
\hline Diabetes, $n$ & $4(23.5 \%)$ & $4(7.5 \%)$ & $8(11.4 \%)$ & 0.0911 \\
\hline Current smoker, $n$ & $1(5.9 \%)$ & $5(9.4 \%)$ & $6(8.6 \%)$ & 1.0000 \\
\hline Alcohol abuse, $n$ & $0(0.0 \%)$ & $1(1.9 \%)$ & $1(1.4 \%)$ & 1.0000 \\
\hline
\end{tabular}

\footnotetext{
${ }^{1}$ Fisher's exact test was used for categorical variables and two sample $t$-test was used for continuous variables. $(p<0.05$ significant). gy $=$ gray.
} 
TABLE 2: Flap type.

\begin{tabular}{|c|c|c|c|}
\hline Type of flap & $\begin{array}{l}\text { Delayed } \\
(N=17)\end{array}$ & $\begin{array}{l}\text { Immediate } \\
(N=53)\end{array}$ & $\begin{array}{c}\text { Total } \\
(N=70)\end{array}$ \\
\hline Free TRAM & $4(23.5 \%)$ & $3(5.7 \%)$ & $7(10.0 \%)$ \\
\hline Pedicle TRAM & $3(17.6 \%)$ & $4(7.5 \%)$ & $7(10.0 \%)$ \\
\hline Free VRAM & $1(5.9 \%)$ & $0(0.0 \%)$ & $1(1.4 \%)$ \\
\hline Pedicle VRAM & $1(5.9 \%)$ & $4(7.5 \%)$ & $5(7.1 \%)$ \\
\hline Pedicle ALT & $3(17.6 \%)$ & $0(0.0 \%)$ & $3(4.3 \%)$ \\
\hline Free ALT & $0(0.0 \%)$ & $3(5.7 \%)$ & $3(4.3 \%)$ \\
\hline Pedicle rectus abdominis & $3(17.6 \%)$ & $0(0.0 \%)$ & $3(4.3 \%)$ \\
\hline Free rectus abdominis & $0(0.0 \%)$ & $2(3.8 \%)$ & $2(2.9 \%)$ \\
\hline Free gracilis & $1(5.9 \%)$ & $0(0.0 \%)$ & $1(1.4 \%)$ \\
\hline Pedicle gracilis & $0(0.0 \%)$ & $1(1.9 \%)$ & $1(1.4 \%)$ \\
\hline Pedicle gastrocnemius & $1(5.9 \%)$ & $5(9.4 \%)$ & $6(8.6 \%)$ \\
\hline STSG & $0(0.0 \%)$ & $5(9.4 \%)$ & $5(7.1 \%)$ \\
\hline FTSG & $0(0.0 \%)$ & $2(3.8 \%)$ & $2(2.9 \%)$ \\
\hline $\begin{array}{l}\text { Pectoralis major/latissimus } \\
\text { dorsi }\end{array}$ & $0(0.0 \%)$ & $1(1.9 \%)$ & $1(1.4 \%)$ \\
\hline DIEP & $0(0.0 \%)$ & $1(1.9 \%)$ & $1(1.4 \%)$ \\
\hline Free serratus anterior & $0(0.0 \%)$ & $1(1.9 \%)$ & $1(1.4 \%)$ \\
\hline TAP & $0(0.0 \%)$ & $1(1.9 \%)$ & $1(1.4 \%)$ \\
\hline Free latissimus & $0(0.0 \%)$ & $3(5.7 \%)$ & $3(4.3 \%)$ \\
\hline Pedicle latissimus & $0(0.0 \%)$ & $2(3.8 \%)$ & $2(2.9 \%)$ \\
\hline Reverse radial forearm & $0(0.0 \%)$ & $3(5.7 \%)$ & $3(4.3 \%)$ \\
\hline Pedicle TFL & $0(0.0 \%)$ & $1(1.9 \%)$ & $1(1.4 \%)$ \\
\hline $\begin{array}{l}\text { Fasciocutaneous } \\
\text { advancement }\end{array}$ & $0(0.0 \%)$ & $1(1.9 \%)$ & $1(1.4 \%)$ \\
\hline Pedicle rectus femoris & $0(0.0 \%)$ & $3(5.7 \%)$ & $3(4.3 \%)$ \\
\hline Sural nerve graft & $0(0.0 \%)$ & $1(1.9 \%)$ & $1(1.4 \%)$ \\
\hline Free fasciocutaneous & $0(0.0 \%)$ & $1(1.9 \%)$ & $1(1.4 \%)$ \\
\hline Pedicle fasciocutaneous & $0(0.0 \%)$ & $1(1.9 \%)$ & $1(1.4 \%)$ \\
\hline Local rotational & $0(0.0 \%)$ & $1(1.9 \%)$ & $1(1.4 \%)$ \\
\hline Free lateral arm & $0(0.0 \%)$ & $2(3.8 \%)$ & $2(2.9 \%)$ \\
\hline Hemisoleus & $0(0.0 \%)$ & $1(1.9 \%)$ & $1(1.4 \%)$ \\
\hline
\end{tabular}

TRAM = transverse rectus abdominis myocutaneous; VRAM = vertical rectus abdominis myocutaneous; STSG = split thickness skin graft; ALT = anterolateral thigh; FTSG = full thickness skin graft; RF = rectus femoris; $\mathrm{TFL}=$ tensor fascia lata; DIEP $=$ deep inferior epigastric artery perforator.

due to the ability to perform wider resection margins knowing that vascularized closure was readily available. While it would be a better study design to perform a prospective trial, ethical standards do not allow for this type of evaluation.

\section{Conclusion}

Delayed reconstruction had a significantly higher incidence of infection and wound dehiscence when compared to immediate reconstruction in the sarcoma limb salvage patient population. Immediate reconstruction may reduce the morbidity associated with this complex reconstruction.
TABLE 3: Outcomes by reconstructive type (univariate).

\begin{tabular}{lccc}
\hline Outcome & $\begin{array}{r}\text { Delayed } \\
(N=17)\end{array}$ & $\begin{array}{c}\text { Immediate } \\
(N=53)\end{array}$ & $p$ value $^{1}$ \\
\hline Infection requiring IV antibiotics & $8(47.1 \%)$ & $5(9.4 \%)$ & 0.0016 \\
Flap failure & $0(0.0 \%)$ & $3(5.7 \%)$ & 1.0000 \\
Partial flap loss & $4(23.5 \%)$ & $5(9.4 \%)$ & 0.2059 \\
Wound dehiscence/drainage & $11(64.7 \%)$ & $16(30.2 \%)$ & 0.0203 \\
Hematoma & $4(23.5 \%)$ & $3(5.7 \%)$ & 0.0542 \\
Seroma & $5(29.4 \%)$ & $11(20.8 \%)$ & 0.5133 \\
\hline
\end{tabular}

${ }^{1} p$ value is from Fisher's exact test.

\section{Competing Interests}

The authors declare that there is no conflict of interests regarding the publication of this paper.

\section{References}

[1] The National Cancer Institute, A Snapshot of Sarcoma, Surveillance, Epidemiology, and End Results (SEER) Program and the National Center for Health Statistics, 2011.

[2] K. K. Curtis, J. B. Ashman, C. P. Beauchamp et al., "Neoadjuvant chemoradiation compared to neoadjuvant radiation alone and surgery alone for stage II and III soft tissue sarcoma of the extremities," Radiation Oncology, vol. 6, no. 1, article 91, 2011.

[3] M. M. Spierer, K. M. Alektiar, M. J. Zelefsky, M. F. Brennan, and P. G. Cordiero, "Tolerance of tissue transfers to adjuvant radiation therapy in primary soft tissue sarcoma of the extremity," International Journal of Radiation Oncology Biology Physics, vol. 56, no. 4, pp. 1112-1116, 2003.

[4] J. M. Arbeit, B. S. Hilaris, and M. F. Brennan, "Wound complications in the multimodality treatment of extremity and superficial truncal sarcomas," Journal of Clinical Oncology, vol. 5, no. 3, pp. 480-488, 1987.

[5] B. G. Peat, R. S. Bell, A. Davis et al., "Wound-healing complications after soft-tissue sarcoma surgery," Plastic and Reconstructive Surgery, vol. 93, no. 5, pp. 980-987, 1994.

[6] K. J. Sanniec, S. Swanson, W. J. Casey, A. Schwartz, L. Bryant, and A. M. Rebecca, "Predictive factors of wound complications after sarcoma resection requiring plastic surgeon involvement," Annals of Plastic Surgery, vol. 71, no. 3, pp. 283-285, 2013.

[7] A. H. Gaur, T. Liu, K. M. Knapp et al., "Infections in children and young adults with bone malignancies undergoing limb-sparing surgery," Cancer, vol. 104, no. 3, pp. 602-610, 2005.

[8] L. Jeys and R. Grimer, "The long-term risks of infection and amputation with limb salvage surgery using endoprostheses," Recent Results in Cancer Research, vol. 179, pp. 75-84, 2009.

[9] R. F. Lohman, A. S. Nabawi, G. P. Reece, R. E. Pollock, and G. R. D. Evans, "Soft tissue sarcoma of the upper extremity: a 5-year experience at two institutions emphasizing the role of soft tissue flap reconstruction," Cancer, vol. 94, no. 8, pp. 2256-2264, 2002.

[10] L. E. Davis and C. W. Ryan, "Preoperative therapy for extremity soft tissue sarcomas," Current Treatment Options in Oncology, vol. 16, no. 6, article 25, 2015.

[11] D. S. Geller, F. J. Hornicek, H. J. Mankin, and K. A. Raskin, "Soft tissue sarcoma resection volume associated with woundhealing complications," Clinical Orthopaedics and Related Research, no. 459, pp. 182-185, 2007. 
[12] E. H. Baldini, M. R. Lapidus, Q. Wang et al., "Predictors for major wound complications following preoperative radiotherapy and surgery for soft-tissue sarcoma of the extremities and trunk: importance of tumor proximity to skin surface," Annals of Surgical Oncology, vol. 20, no. 5, pp. 1494-1499, 2013.

[13] W. J. Barwick, J. A. Goldberg, S. P. Scully, and J. M. Harrelson, "Vascularized tissue transfer for closure of irradiated wounds after soft tissue sarcoma resection," Annals of Surgery, vol. 216, no. 5, pp. 591-595, 1992. 


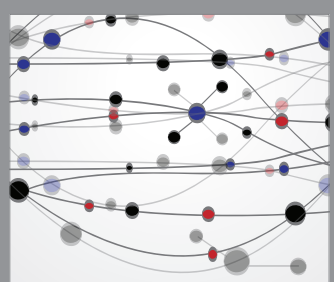

The Scientific World Journal
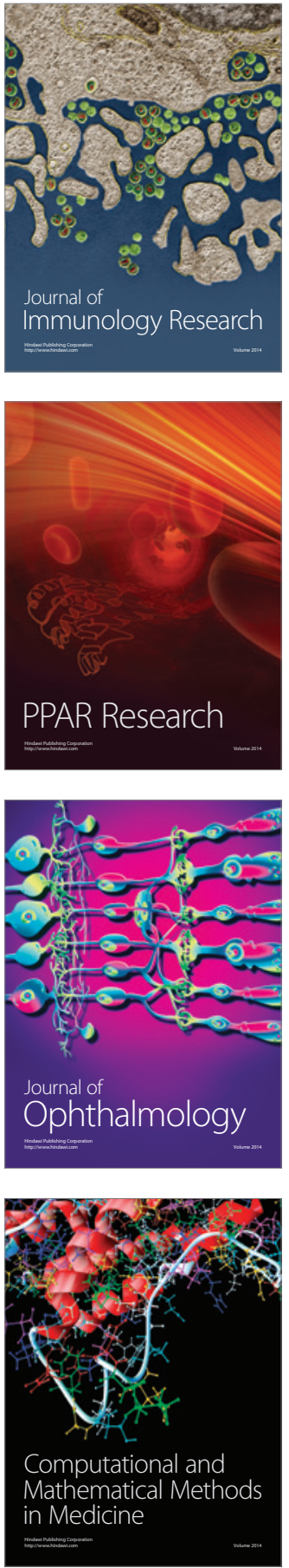

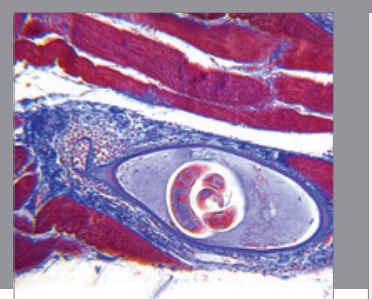

Gastroenterology Research and Practice

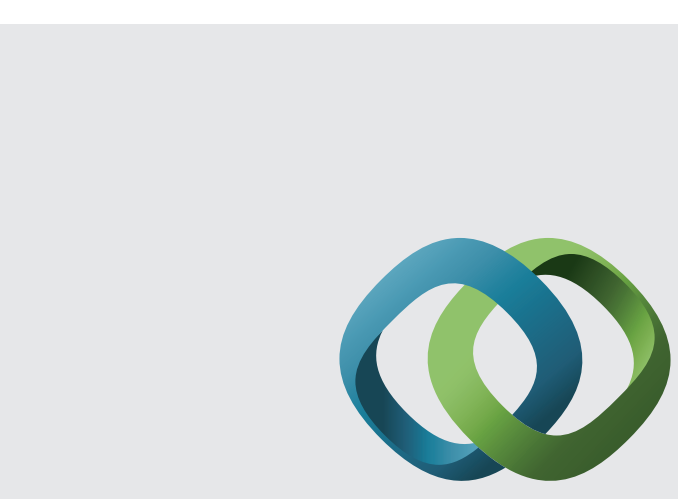

\section{Hindawi}

Submit your manuscripts at

http://www.hindawi.com
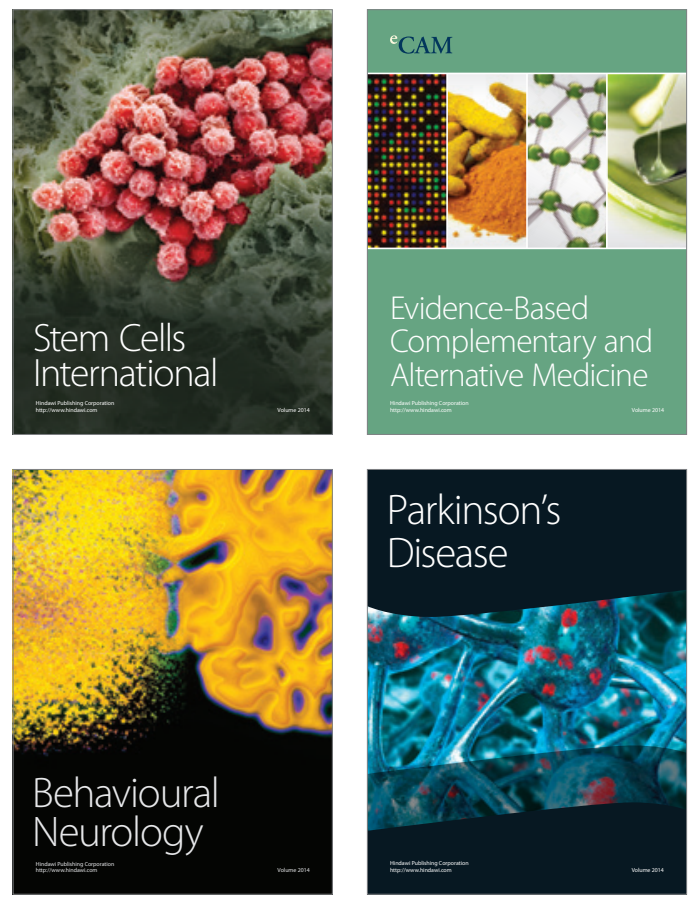
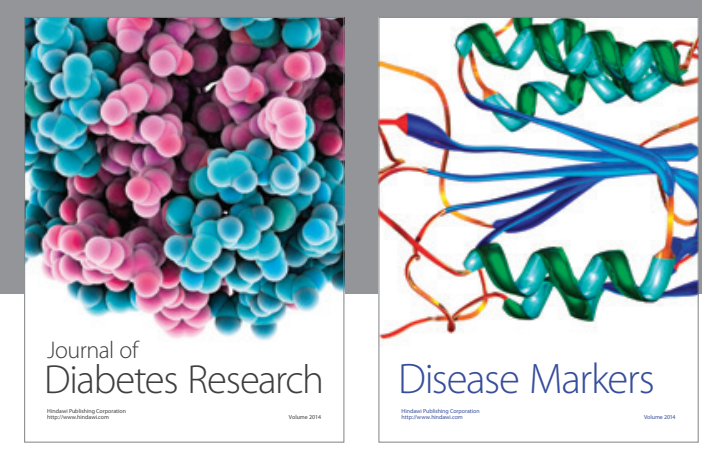

Disease Markers
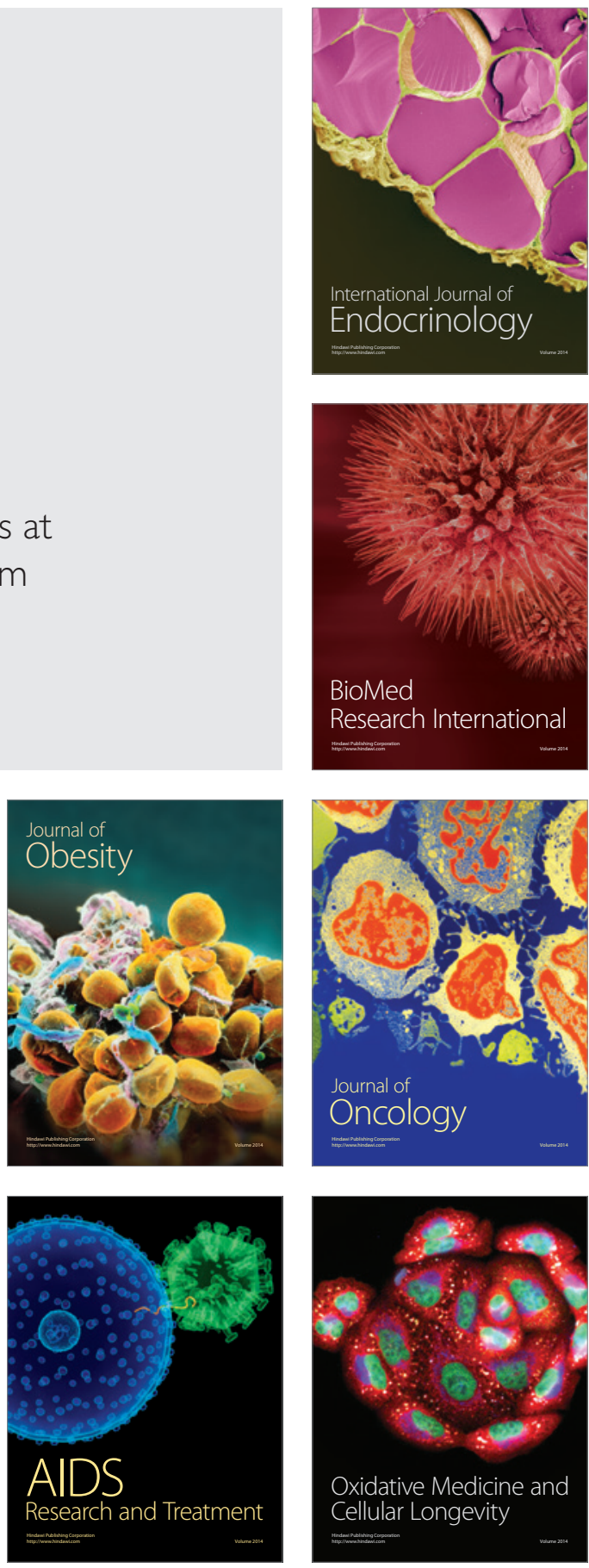